\title{
REFERENCES
}

1. W. Engel, Ganze Cremona-Transformationen von Primzahlgrad in der Ebene, Math. Ann. vol. 136 (1958) pp. 319-325.

2. H. W. E. Jung, Einführung in die Theorie der algebraischen Functionen zweier Veränderlicher, Berlin, Akademie Verlag, 1951, p. 61.

3. P. Samuel, Singularitês des variêtés algébriques, Bull. Soc. Math. France vol. 79 (1951) pp. 121-129.

University of California, Berkeley

TEChNion, Haifa, IsRaEL

\section{ON PROJECTIVE MODULES OVER SEMI-HEREDITARY RINGS}

\section{FELIX ALBRECHT}

This note contains a proof of the following

THEOREM. Each projective module $P$ over a (one-sided) semi-hereditary ring $\Lambda$ is a direct sum of modules, each of which is isomorphic with a finitely generated ideal of $\Lambda$.

This theorem, already known for finitely generated projective modules [1, I, Proposition 6.1], has been recently proved for arbitrary projective modules over commutative semi-hereditary rings by $\mathrm{I}$. Kaplansky [2], who raised the problem of extending it to the noncommutative case.

We recall two results due to Kaplansky:

Any projective module (over an arbitrary ring) is a direct sum of countably generated modules [2, Theorem 1$]$.

If any direct summand $N$ of a countably generated module $M$ is such that each element of $N$ is contained in a finitely generated direct summand, then $M$ is a direct sum of finitely generated modules [2, Lemma 1].

According to these results, it is sufficient to prove the following proposition:

Each element of the module $P$ is contained in a finitely generated direct summand of $P$.

Let $F=P \oplus Q$ be a free module and $x$ be an arbitrary element of $P$. Let $x=\lambda_{1} x_{1}+\cdots+\lambda_{n} x_{n}$ be a representation of the element $x$ in some base for the free module $F$ and let $G$ denote the free submodule

Received by the editors June 28, 1960. 
of $F$, generated by the elements $x_{1}, \cdots, x_{n}$. Consider the homomorphism $\phi: G \rightarrow Q$, induced by the projection of $F$ onto $Q$. Obviously, $\operatorname{Ker} \phi=G \cap P \ni x$ and $G / G \cap P$ is isomorphic to $\operatorname{Im} \phi$. Since $\operatorname{Im} \phi$ is a finitely generated submodule of the projective module $Q$ and the ring $\Lambda$ is semi-hereditary, $\operatorname{Im} \phi$ and therefore $G / G \cap P$ is projective. It follows that $G \cap P$ is a direct summand of $G$ and hence finitely generated. Since $G$ is a direct summand of $F$, the module $G \cap P$ is also a direct summand of $F$ and, being contained in $P$, a direct summand of $P$.

\section{REFERENCES}

1. H. Cartan and S. Eilenberg, Homological algebra, Princeton University Press, 1956.

2. I. Kaplansky, Projective modules, Ann. of Math. vol. 68 (1958) pp. 372-377.

Institute of Mathematics, R.P.R. Academy, Bucharest, Rumania 